\title{
ROLE OF CDK12 IN REGULATION OF DNA DAMAGE RESPONSE IN OVARIAN CANCER CELLS
}

\section{Jaroslav Klát ${ }^{1}$, Marta Dzimková ${ }^{2}$, Hana Paculova ${ }^{2}$, Sylva Bajsová ${ }^{1}$, Jiri Kohoutek ${ }^{2}$}

${ }^{1}$ Department of Obstetrics and Gynecology, University Hospital, Ostrava, Czech Republic

${ }^{2}$ Department of Chemistry and Toxicology, Veterinary Research Institute, Brno, Czech Republic

\section{Abstract}

The DNA-damage-response (DDR) pathway is a cellular mechanism which has evolved to protect cellular integrity by detection and repair of DNA lesions. Previously, our group and others demonstrated that the cyclindependent kinase 12 (CDK12) maintains genome stability via regulation of transcription of DDR genes, specifically, BRCA1, RAD51 and others. Importantly, down-regulation of the CDK12 caused induction of the 53BP1 and YH2AX foci and accumulation of cells in the G2-M phase of the cell cycle. Since various microRNA (miRNA) are situated within coding genes, we hypothesized that expression of some of them might be also affected by CDK12 depletion. Therefore, we conducted a pilot study focused on identification of candidate miRNAs that might be significantly altered in CDK12 deficient cells. Indeed, downregulation of CDK12 protein level led to aberrant expression of several miRNAs. Among studied miRNAs, the level of miR-152 was significantly elevated. By using predictive algorithm, several proteins that might be specifically targeted by miR-152 were examined. We confirmed that upregulated expression of miR-152 leads to decreased expression of DNMT1, RICTOR and MET proteins which are often found deregulated in rather wide spectrum of oncogenic diseases. Defects in methylation of miR-152 has been observed in several cancers and studies have proven an on/of loop between expressions of DNMT1 and miR-152. We speculate that CDK12 participates in DDR machinery by two distinct mechanisms, either by orchestrating transcription of DDR genes or by stabilization of DNMT1 protein by blocking expression of miR-152 targeting DNMT1.

We confirmed that upregulated expression of miR-152 leads to decreased expression of DNMT1, RICTOR and MET proteins which are often found deregulated in rather wide spectrum of oncogenic diseases. Defects in methylation of miR-152 has been observed in endometrial and breast cancer and studies have proven an on/of loop between expressions of DNMT1 and miR-152 who acts as antagonist to each other. Over expression of RICTOR has been also documented in cases of endometrial cancer and in esophageal cancer along with upregulation of MET promoting angiogenesis and earlier incidency of metastasis. We speculate that CDK12 participates in DDR machinery by two distinct mechanisms, either by orchestrating transcription of DDR genes or by stabilization of DNMT1 protein by blocking expression of miR-152 targeting DNMT1. Currently, we aim to determine the precise mechanism underlying the role of CDK12 in this process.

\section{Results}

A)

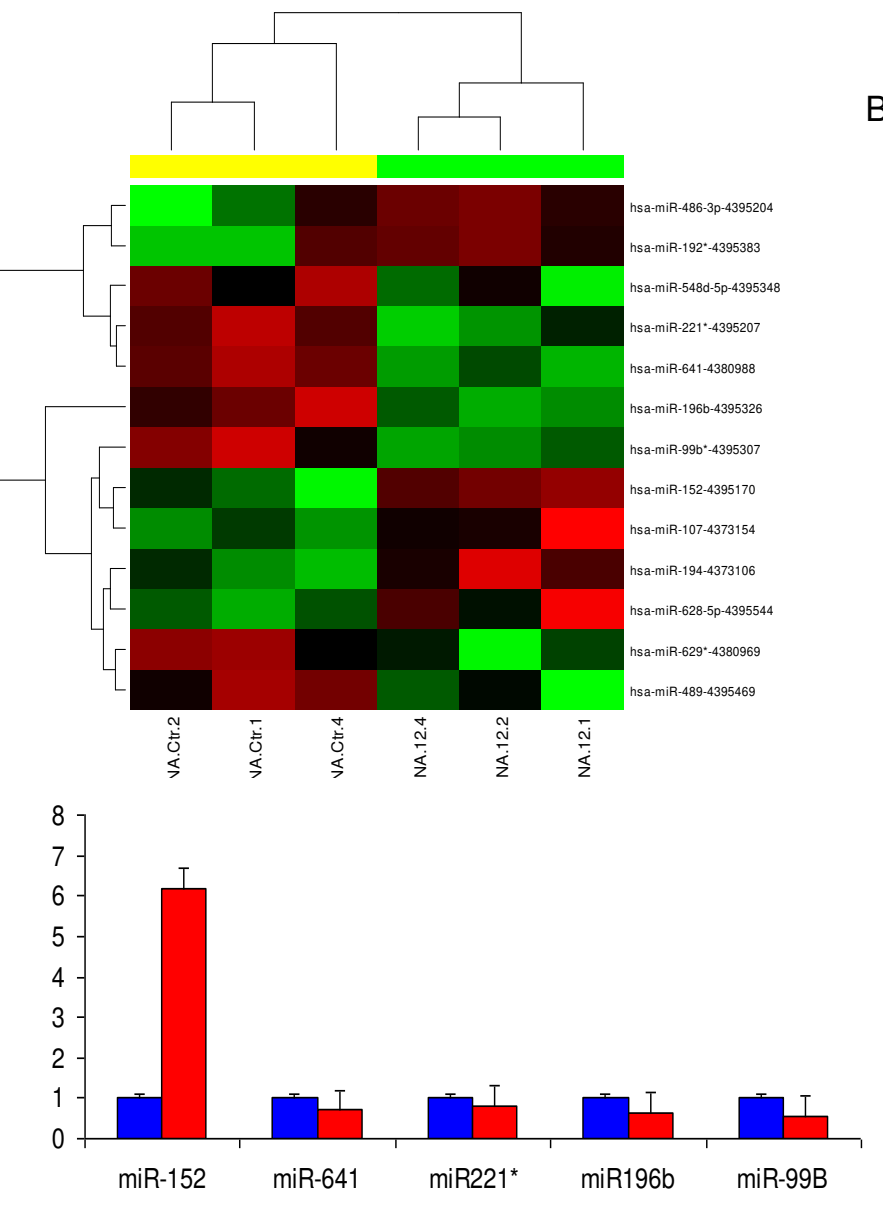

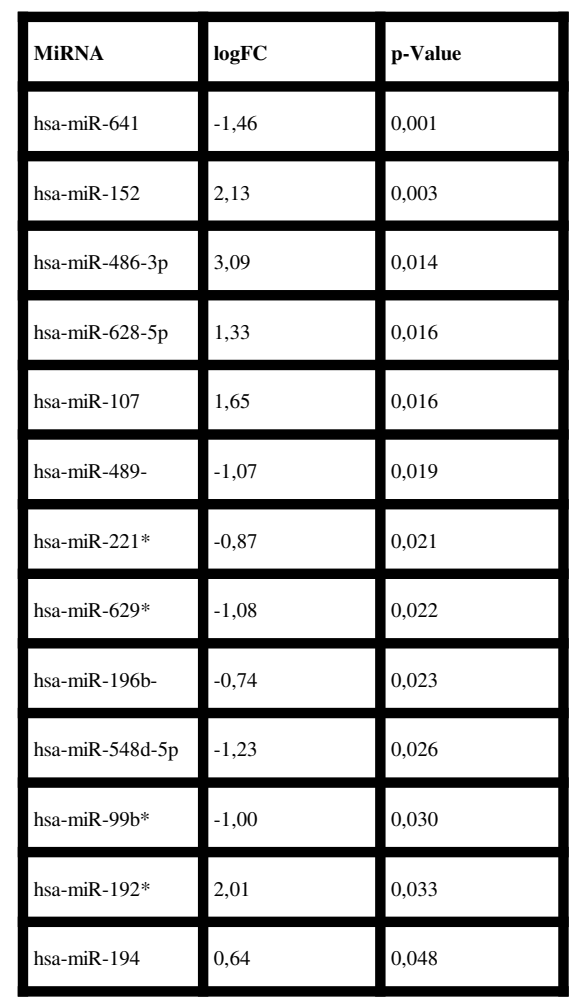

Figure 1: A) Dendrogram and heat map depicting levels of 13 miRNAs in control cells and cells with depleted CDK12. Cells in yellow column are controls cells marked as NA.Ctr.1, Ctr.2 and Ctr.4, and cells with depleted CDK12 are in green column marked as NA.12.1,12.2 and 12.4. Heat map represents up/down regulation of candidate miRNAs, where green color represents downregulaion of given miRNA while red color represents upreulation of given miRNA.

B) List of putative miRNAs with different expression levels detected in control cells and in cell with CDK12 silenced using siRNA. HCT116 cell line was used for this purpose.

C) Levels of miRNAs determined using RT-qPCR. From miRNAs listed in table B), we choose 5 putative miRNAs, to evaluate their levels under various condition. Blue column represents control cells, red column cells with depleted CKD12. Again, HCT116 cell line was used.

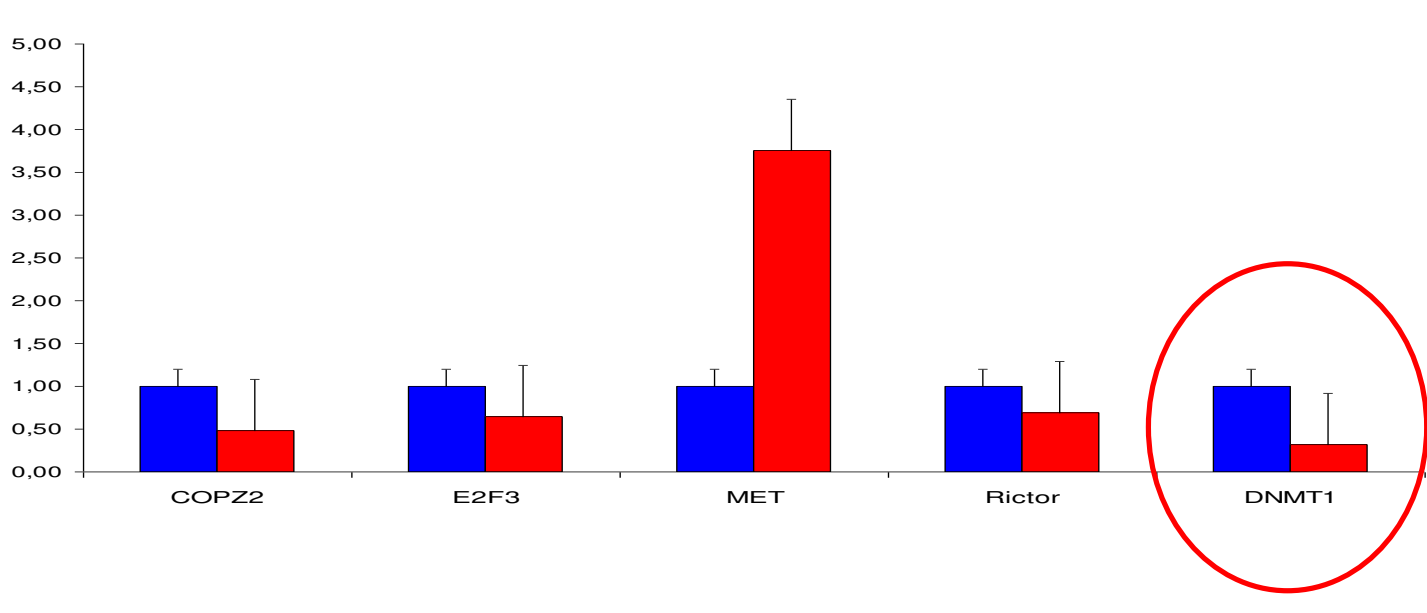

Figure 2: Graph depicts relative levels of mRNA of particular proteins in HCT116 cells in the presence or absence of CDK12. RT and q-PCR were performed after 72 hours treatment with siRNAs. Blue columns are control, untreated cells, red columns are cells treated with CDK12 SiRNA

Since miR-152 shoved enhanced level after CDK12 depletion, we decided to focus further on its function and targets. There are known genes which are targeted by miR-152, among them COPZ2, E2F3, MET, RICTOR and DNMT1. These genes also seems to play significant role during tumor development and progression. Thus the effect of miR-152, induced by depletion CDK12, on mRNA level of above mentioned genes was evaluated. Further we decided o evaluate CDK12 depletion on other cell lines, such as SKBR3 and SKOV3, with keeping HCT116 as reference line. Initially we did Westernblos screens for all 5 genes, but with COPZ2 and E2F3 being inconsistent we focused mostly on DNMT1

We then tested our hypothesis about interactions between the target genes, miR152 and CDK12 by depleting CDK12 using siRNA, inhibiting miR152 using anti-sense miRNA, treating cells with miR152 mimic RNA, combination of si RNA depletion of CDK12 and miR152 antisense, or by using novel CDK12 inhibitor THZ 531 and its combination with anti-sense miRNA.

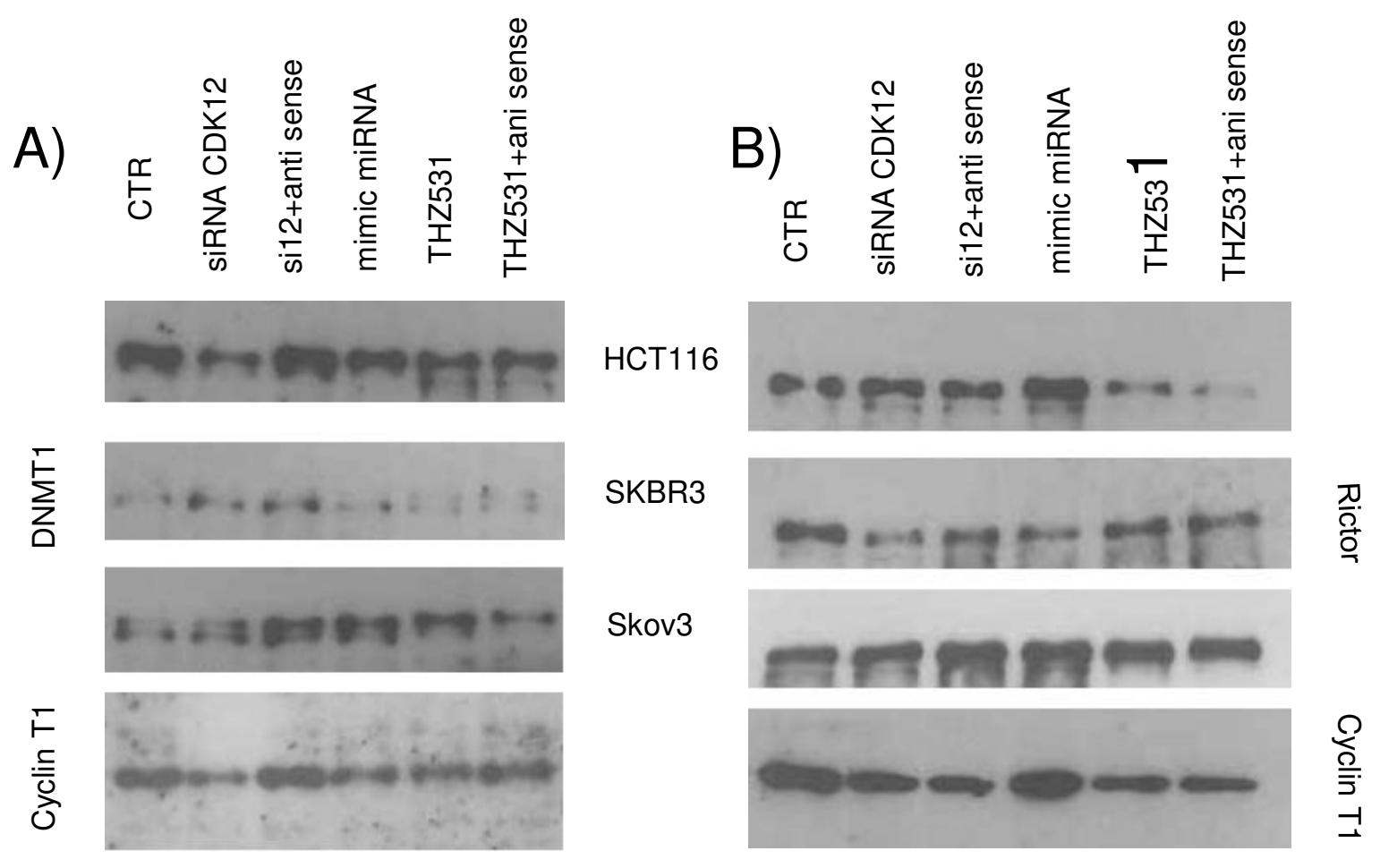

Figure3: Western blot representation target protein levels in 3 cell lines under various treatment. Each treatment lasted for 72 hours and standard grow media were used according to each cell line needs.

A) Levels of DNMT1 where a decrease is visible after depletion of CDK12, and restoration after treatment with anti-sense miRNA implies, that this protein is regulated ba both CDK12 and miR152 sudgesting a close relation between these two.

B) Levels of Rictor, where there is slight decrease after depletion of CDK12, but more marquant effect is seen after treatment with the CDK inhibitor itself, thus proposing, it is not

co-regulated with miR152

\section{Summary}

CDK12 regulates transcription of specific protein coding genes, such as genes involved in DNA-damage repair (DDR), by phosphorylation of RNA polymerase II. Since majority of ncRNA, among them miRNAs, are located within protein coding genes, we were intrigued by a simple question, whether depletion of CDK12 will affect transcription/expression of particular miRNAs?

We found out, that some genes are indeed affected a nd also that CDK12 and miR152 might act concordantly with other regulational mechanisms.

In summary, besides its known role in DDR pathway, CDK12 also seems to participate in the transcription/expression of unique miRNAs and thus impacts process of tumorigenesis. 